sclera overlying the cyst and the cyst is then evacuated, progress seems to be uniformly good." It would appear, however, to be a safer procedure in all cases and more especially if the cyst has ruptured, to place a ring of diathermy surface applications over the retina all along the inner periphery of the cyst, in addition to those over the cyst itself, before puncturing the latter. In this way the inter-retinal space should become securely sealed off and the risk of a spreading detachment obviated, even if through stretching of the cyst wall snug apposition of the latter to the underlying choroid is prevented.

\title{
REPERENCES
}

1. RIDLEY (1935).-Brit. Jl. Ophthal., Vol. XIX, p. 101.

2. GouldeN (1935).-Trans. Ophthal. Soc. U.K., Vol. LV,.p. 407.

\section{CONGENITAL PARALYSIS OF BOTH EXTERNAL RECTI TREATED BY TRANSPLANTATION OF EYE MUSCLES*}

\author{
BY \\ JOHN A. MAGNUS \\ YORK
}

Miss P. W., aged 12 years. This child has a gross spinal deformity (kyphosis, scoliosis and torticollis) from birth. $\mathrm{X}$-ray report at the age of 3 years (1933): Spina bifida occulta, involving all the cervical vertebrae and some of the upper dorsal, left-winged scapula.

I saw the child first on October 28, 1942, when I diagnosed: Bilateral abducens paralysis, right marked hyperphoria due to overacting right inferior oblique. (See Fig. 1). The vision is $6 / 6$ in each eye, and the fundi are normal. The child has no binocular vision. She suppresses the right image.

On January 16, 1943, the left eye was operated upon under a general anaesthetic. I performed a recession of the left internal rectus $(5 \mathrm{mms}$.) which was very contracted and thick. Following O'Connor's technique, I made a semi-circular incision in the conjunctiva around the temporal half of the cornea, about $10 \mathrm{mms}$. away from the limbus, the incision extending from "12 to 6 oclock." I exposed the superior and inferior recti, and split the tendons longitudinally for about $12 \mathrm{mms}$. with an old Graefe knife. A double-armed catgut suture was then run through the outer halves of the superior and inferior recti, and firmly tied. The segments were now dissected off their insertions and sewn beneath the

- Received for publication, January 19, 1944. 
external rectus, 'which was slightly advanced at the' same time. (See Figs. 2 and 3).

I was very astonished to find a thick, healthy-looking external rectus muscle, which was at least twice as thick as normal, and appeared to come from below, running obliquely upwards, the

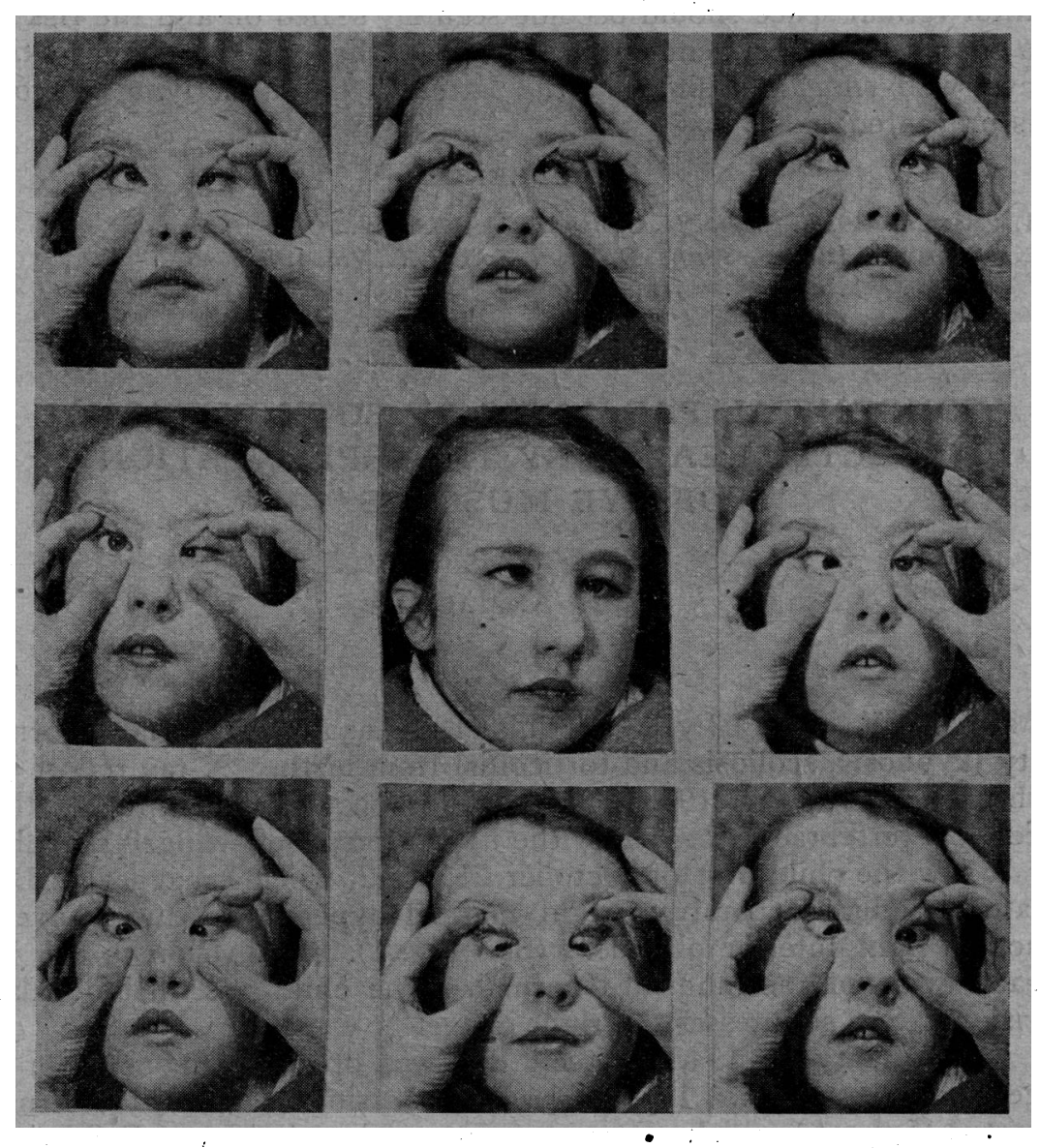

FIG. 1.-BEFORE OPERATION.

insertion being slightly higher than normal. The superior and inferior recti were much thinner than the external rectus, and more poorly developed than normal.

The right eye was operated upon on January 23, 1943. A similar recession and transplantation were carried out, as well as a myectomy of the right inferior oblique, which was reached from the lower

Congenital paralysis of both external recti treated by transplantation Congenital paraly
of eye muscles. 
fornix of the conjunctiva, excising about $10 \mathrm{mms}$. of the muscle. The appearance of the right eye muscles was very similar to that found on the left side. The external rectus was again much thicker than normal, but more or less in the correct anatomical position.

After these operations, both eyes looked cosmetically straight, but the left eye showed "a slight hyperphoria. There was, however,

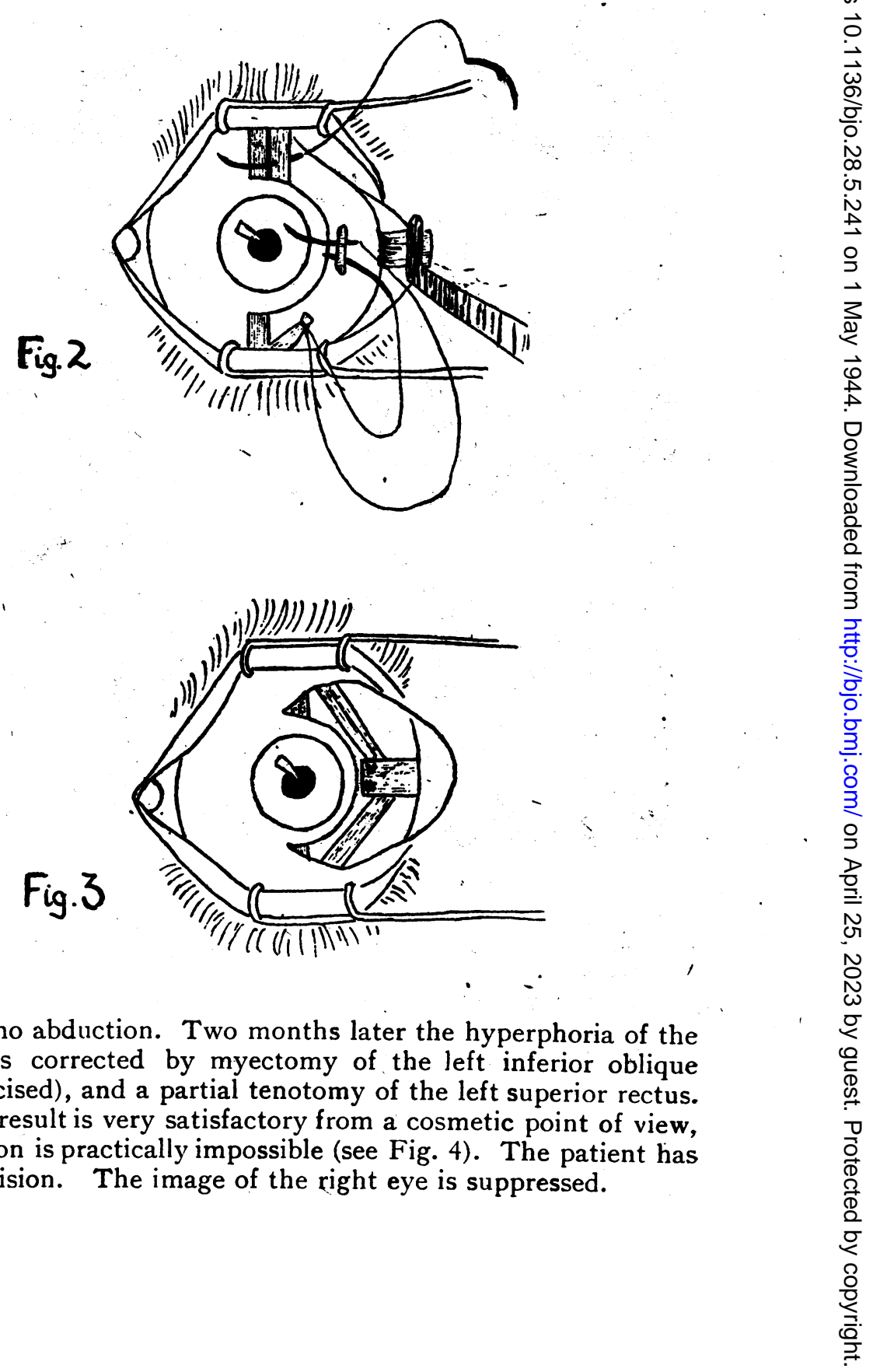

practically no abduction. Two months later the hyperphoria of the left eye was corrected by myectomy of the left inferior oblique (6 mms. excised), and a partial tenotomy of the left superior rectus.

The end result is very satisfactory from a cosmetic point of view, but abduction is practically impossible (see Fig. 4). The patient has no double vision. The image of the right eye is suppressed. 

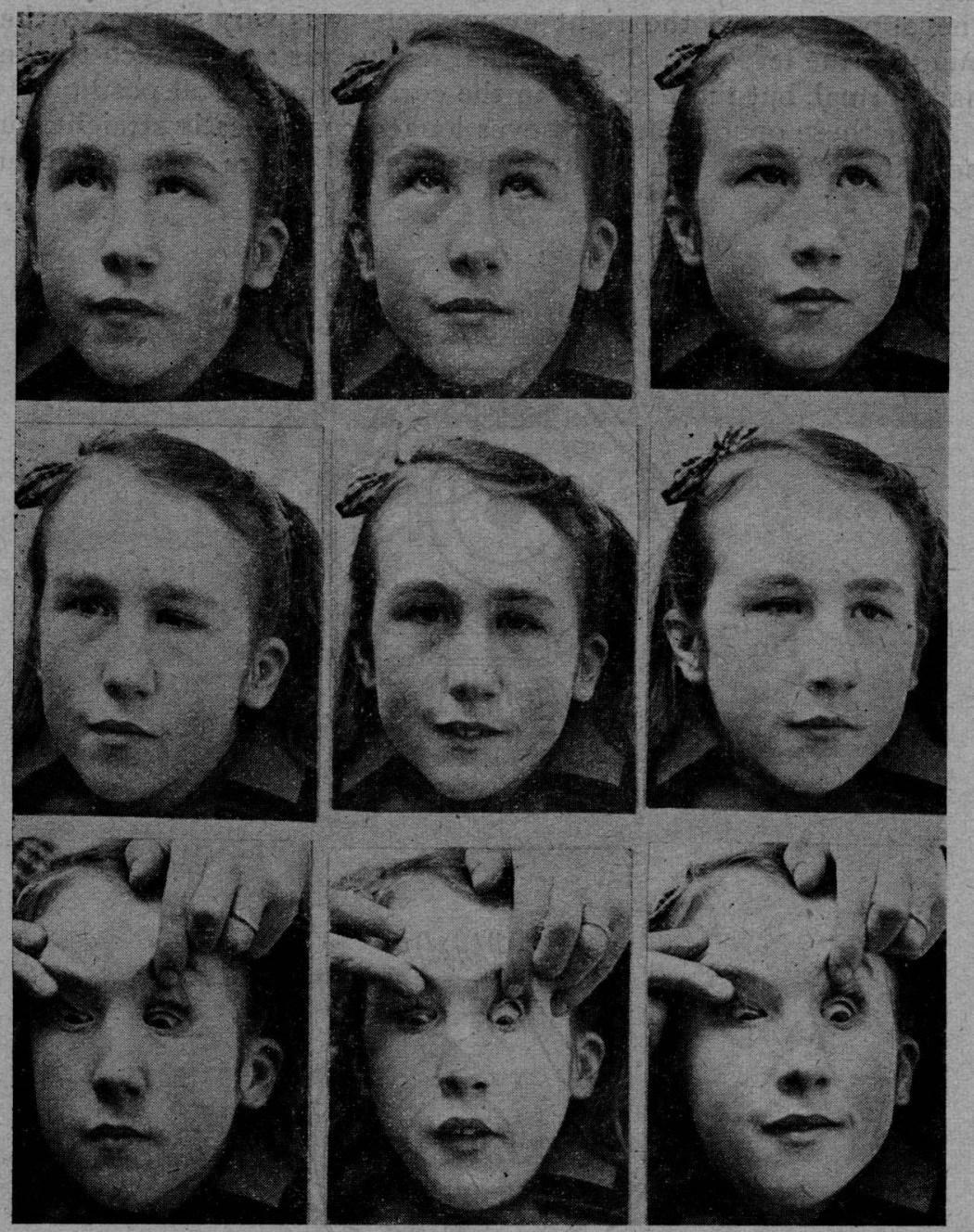

Fig. 4.-After all Four Óperations. 


\title{
Comments
}

A case of bilateral abducens paralysis is reported, which was treated by transplantation of the outer halves of the superior and inferior recti to the insertion of the paralysed external rectus. This latter muscle was much thicker than normal, and showed in the left eye an abnormal anatomical position.

I thought that a possible explanation for the abnormal thickness of the external rectus (one would have expected a thin, frail muscle in a congenital paralysis of the 6 th-nerve), might be the innervation of the external rectus by the 3 rd nerve instead, of the 6 th. (The internal and external recti always contracting at the same time). But Miss Ida Mann, of Oxford, informed me that this is embryalogically impossible, because the 6 th nerve is the motor nerve of the 3 rd head cavity, and the 3 rd nerve of the 1 st head cavity. One cannot imagine very well the segmental nerve supply jumping two sections, nor does it seem likely that the muscle fibres themselves have crossed over in the cone of condensation of mesoderm, from which they all come.

I am therefore at a loss as to the explanation of this case, unless it is caused by the spina bifida occulta of the cervical vertebrae, nor why the transplantation did not bring about the expected abduction of the eyes.

My thanks are due to Miss Mann for her kind advice.

\section{UNILATERAL FOLLICULAR CONJUNCTIVITIS DUE TO MOLLUSCUM CONTAGIOSUM*}

BY

\author{
JOHN A. MAGNUS \\ YOR K
}

THE rarity of this disease in this country justifies the publication of the following case :-

Mrs. F. E. W., aged 53 years. I saw the patient first on March 16, 1943, when she had an acute conjunctivitis and superficial keratitis of the right eye, which had developed after influenza. She was treated along the usual lines with guttae sodium sulphacetamide 30 per cent., boracic lotion and ung. merthiolate, 1 in 5,000, aolan injections and vitamin $\mathrm{C}$, but the condition did not clear up during the next two months.

Various investigations were carried out :-X-ray sinuses, N.A.D. ; Ear, nose and throat examination, N.A.D.; Mantoux, negative; Culture, sterile.

As they did not reveal the cause, the local treatment was changed, and guttae neoprotosil 10 per cent., and sol. hydrarg. oxycyanide, 1 in 5,000 , given. The conjunctiva was painted with the alum

\footnotetext{
* Received for publication January 3, 1944.
} 